\title{
Terminology Guidelines for Serotaxonomic Studies Using Immunodiffusion and Immunoelectrophoresis
}

\author{
SOTIROS D. CHAPARAS, ${ }^{1 *}$ ARNE LIND, ${ }^{2}+$ ÖRJAN OUCHTERLONY, ${ }^{2}$ AND MALIN RIDELL ${ }^{2}$ \\ National Center for Drugs and Biologics, Food and Drug Administration, Bethesda, Maryland 20205, ${ }^{1}$ and \\ Department of Medical Microbiology, University of Goteborg, Goteborg, Sweden ${ }^{2}$
}

We propose guidelines for terminology for the immunodiffusion and immunoelectrophoresis methods used in serotaxonomy. These guidelines were prepared at the request of the International Working Group on Mycobacterial Taxonomy, which endorsed them in 1981.

The range of taxonomic tests used today is wide and covers different scientific domains. Therefore, taxonomists must be familiar with a variety of analytical techniques. Handbooks and other publications generally provide necessary information concerning methodology. However, taxonomists may encounter terms that lack precision, and this may lead to misinterpretation of the results obtained by different investigators. One remedy for this problem would be an improved and standardized terminology acceptable to the investigators concerned. In this paper we present a proposal for terminology guidelines for the methods used for immunodiffusion and immunoelectrophoresis, a group of methods commonly used in serotaxonomy.

Immunodiffusion and immunoelectrophoresis techniques were introduced in the 1940s and 1950s. Since these techniques are comparatively simple and have a high resolving power, they were soon used for analyses of the antigenic mosaic of bacteria, including serotaxonomical aspects. The originators of the techniques tried to create a simple descriptive technical terminology, but their attempts were only partially successful. Later, several modifications of the original immunodiffusion and immunoelectrophoresis techniques were described, and new methods within the same area of seroanalytical technology were developed. In connection with these advances problems in terminology arose; laboratory jargon and personal names for certain techniques came into common use. Such terminology may suffice for referring to a methodological principle; however, it may be too ambiguous for proper identification of a particular analytical test. Also, the terminology used for the basic phenomena observed when analyses are performed by various immunodiffusion and immunoelectrophoresis techniques has become less and less uniform. This trend is regrettable, as it could lead to erroneous interpretations of analytical results.

† Author to whom reprint requests should be addressed.
The International Working Group of Mycobacterial Taxonomy (IWGMT) has organized a number of cooperative systematic studies involving many laboratories in different countries. Although these studies have been mainly based on numerical taxonomy, many of them have also included serotaxonomic investigations in which agglutination and precipitation (including immunodiffusion and immunoelectrophoresis) techniques were used. IWGMT members concerned with the latter types of techniques realize the need for a standardized nomenclature, as well as an inventory of the technical synonyms in common use. In 1979 the IWGMT decided to act on this matter, and we were requested to prepare guidelines for terminology to be used for immunodiffusion and immunoelectrophoretic methods in mycobacterial taxonomy. At a meeting in 1981, the IWGMT endorsed our guidelines on terminology. The guidelines agreed upon may also be useful to investigators outside the IWGMT who do not have access to its informal communication, Forum Mycobacteriorum. Therefore, in this paper we present the IWGMT terminology guidelines to a wider circle of workers in systematic bacteriology.

\section{TERMINOLOGY}

Abbreviations. The following abbreviations are used below: $a$ and $b$, monospecific unrelated antigens; $a$ and $a_{1}$, monospecific related antigens;(ab),bispecific antigen; A and B, antibodies corresponding to antigens $a$ and $b$, respectively; $A$, antibodies that react with antigen a but not with antigen $a_{1} ; A_{1}$, antibodies that react with both antigen a and antigen $a_{1}$.

\section{Materials}

(i) Materials for immunization Immunogen Complete adjuvant (Freund) Incomplete adjuvant Other adjuvants (e.g., alum)

(ii) Antigen and antigen preparations for 
immunodiffusion and immunoelectrophoresis Antigen (or precipitinogen or antigen reactant)

Monospecific antigen (antigen particle having determinants of only one specificity (e.g., a)

Multispecific antigen (antigen particle having determinants of two or more different specificities) (e.g., ab)

Antigen preparation and reference antigen preparations (when used, for example, in a reference system)

(iii) Antibody and antiserum

Antibody (or precipitin or antibody reactant Polyclonal (multiclonal) antibody

Monoclonal (hybridomal myeloma) antibody

Antiserum and reference antisera (when used, for example, in a reference system)

Multispecific antiserum (e.g., A + B)

Monospecific antiserum (e.g., A)

(iv) Serological systems and reference serological systems (implying the use of reference antigen and reference antiserum)

Homologous system (system consisting of antigen preparation and antiserum obtained by immunization with the same preparation) Heterologous system (system consisting of antigen preparation and antiserum obtained by immunization with a different material) Simple system (e.g., $a=A$ )

Complex system (e.g., $a+a_{1}=A+A_{1}$ )

Multiple system (e.g., $a+b=A+B$ )

System with multispecific antigen (e.g., (ab) $=A+B)$

Note that serological systems are called balanced when antigen and antibody reactants are at equivalent concentrations and produce optimal or maximal precipitation; when they are not, the systems are unbalanced.

Methods (For technical details, see references 1 through 3.)

(i) Immunodiffusion (ID)

Simple diffusion in one dimension (e.g., tube technique [Oudin procedure])

Simple diffusion in two dimensions (e.g., plate technique [single radial immunodiffusion or Mancini procedure])

Double diffusion in one dimension (e.g., tube technique [Oakley-Fulthorpe procedure])

Double diffusion in two dimensions (e.g., plate technique [Ouchterlony procedure]) and various comparative double diffusion and comparative inhibition plate techniques [e.g., Nilsson procedure]

(ii) Immunoelectrophoresis (IE)

Immunoelectrophoresis without immunodiffusion (electroimmunoanalysis)
Electrophoresis in one dimension (1D-IE)

Electrophoresis of antigen or antibody reactant

Electrophoresis of antigen into an antibody-containing gel

Rocket immunoelectrophoresis (electroimmunoassay by the method of Laurell) (circular well application) and various fused rocket immunoelectrophoresis procedures (adjacent wells)

Line immunoelectrophoresis (rectangular well application) and various fused line immunoelectrophoresis procedures (adjacent wells)

Electrophoresis of antibody into an antigen-containing gel (reversed procedure)

Reversed rocket immunoelectrophoresis

Reversed line immunoelectrophoresis

Electrophoresis of antigen and antibody reactants

Countercurrent electrophoresis (CEP) (simultaneous counterelectrophoresis of antigen and antibody in gel)

Electrophoresis in two dimensions (crossed immunoelectrophoresis, 2D-IE)

Regular crossed immunoelectrophoresis (first run, electrophoresis of a single antigen preparation into a neutral gel; second run, electrophoresis [perpendicular to the previous run] of the antigen into an antibody-containing gel) Tandem crossed immunoelectrophoresis (first run, electrophoresis of two or more antigen preparations in separate wells placed in a single file into a neutral gel; second run, electrophoresis [perpendicular to the previous run] of the antigens into an antibody-containing gel).

Note that the techniques mentioned above can be modified by interposing a third antigen- or antibody-containing (intermediate) gel between the original antigen-containing gel and the antibody-containing gel.

Immunoelectrophoresis with immunodiffusion

Electrophoresis in one dimension

Conventional immunoelectrophoresis

(e.g., macrotechnique [Grabar-Williams procedure] and microtechnique [Scheidegger procedure]

Electrophoresis of antigen preparation followed by double diffusion of antigen and antibody 


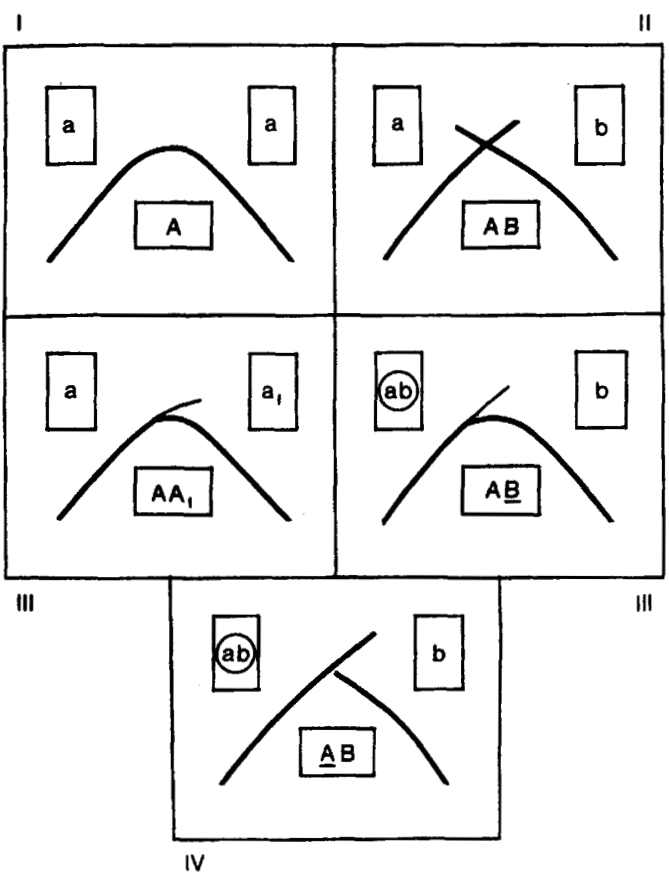

FIG. 1. Basic interaction phenomena in comparative double diffusion (reaction types I through IV). For explanation of abbreviations, see text. The underlining indicates antibodies that have a leading edge during diffusion.

Electrophoresis of antiserum followed by double diffusion of antibody and antigen

Note that the techniques mentioned above can be modified by adding supplementary wells of antigen preparations of antisera to the ordinary double-diffusion arrangement (comparative immunoelectrophoresis).

TABLE 1. Description of reaction types I through IV for comparative double diffusion

\begin{tabular}{cccc}
\hline $\begin{array}{c}\text { Reaction } \\
\text { type }\end{array}$ & \multicolumn{1}{c}{ Description } & Interference & Deviation \\
\hline I & $\begin{array}{c}\text { Reaction of complete } \\
\text { fusion } \\
\text { II }\end{array}$ & Yes & Yes \\
Reaction of \\
noninteraction & $\begin{array}{c}\text { Reaction of partial } \\
\text { fusion (true spur) }\end{array}$ & Yes & Yes \\
IV & Reaction of inhibition & Yes & No \\
\hline
\end{tabular}

a Reaction types also can be used for reactions observed in other comparative techniques (e.g., comparative immunoelectrophoresis).

\section{Results}

(i) Description of results

Precipitation pattern

Precipitate and precipitation line (band)

Basic interaction phenomena in comparative double diffusion (Fig. 1 and Table 1) Spur phenomena

True spur (reaction type III in immunodiffusion; apical spur in rocket immunoelectrophoresis)

False spur (distortion of reaction type I)

(ii) Designation of results

Letter system: aA, bB, cC, etc. (a, precipitinogen; A, precipitin; aA, precipitate)

Number system: 1, 2, 3, etc. (numbers indicate precipitinogen, precipitin, and precipitate)

(iii) Interpretation of results

Qualitative analyses: interpretation of antigen comparison

Reaction type I: identical antigenic determinants (provided an adequate antiserum is used)

Reaction type II: no serological relationship

Reaction types III and IV: serological relationship (partial identity) but antigens compared not completely identical

Qualitative analyses: interpretation of antibody comparison

Reaction type I: (a) antibody specificity identical (provided that monospecific antigen particles are used); (b) antibody specificity not necessarily identical (antibody reacting with its corresponding determinant on a multispecific antigen particle)

Reaction type II: antibody specificity different

Quantitative analyses (for example, single radial immunodiffusion [size of circular precipitate area correlates with concentration] and rocket immunoelectrophoresis [height of precipitate peak correlates with concentration])

\section{LITERATURE CITED}

1. Axelsen, N. H., J. Kroll, and B. Weeke. 1973. A manual of quantitative immunoelectrophoresis. Methods and applications. Universitetsforlaget, Oslo.

2. Crowle, A. J. 1973. Immunodiffusion, 2nd ed. Academic Press, Inc., New York.

3. Ouchterlony, O., and L.-A. Nilsson. 1978. Immunodiffusion and immunoelectrophoresis, p. 19.1-19.44. In D. M. Weir (ed.), Handbook of experimental immunology, 3rd ed. Blackwell Scientific Publications, Oxford. 